\title{
Capacidad de predicción de esfuerzos de conformado de materiales metálicos. Aplicación a la aleación Ti-6Al-4V $\mathrm{V}^{(\bullet)}$
}

\author{
I. Rieiro ${ }^{(*)}$, A. Martínez ${ }^{(*)}$ y M. Carsi ${ }^{(*)}$
}

\begin{abstract}
Resumen Se describe el uso y explotación de un algoritmo integrado para el ajuste de la ecuación de Garofalo a los resultados de ensayos experimentales de torsión y de tracción, y su capacidad de predecir valores para las variables de conformado, fuera de los rangos de trabajo de los ensayos; se analiza la verosimilitud del ajuste y de las predicciones realizadas. Se ha aplicado el método a los resultados de ensayos de torsión y de tracción para la aleación Ti-6Al-4V.
\end{abstract}

Palabras clave: Ecuación de Garofalo. Predicción de fluencia. Deformación plástica. Análisis numérico. Análisis estadístico.

\section{Predictive capability for forming work of metallic materials. Application to a Ti-6Al-4V alloy}

\begin{abstract}
The explotation and the use of an integrated algorithm for the Garofalo's equation fitting to the experimentals results of torsion and tension tests are described. Its capability for predicting values of the forming work variables, out of the work experimentals ranges is described. The likelihood of the fitting and the predictions are analized. This method has been applied to the experimental results of the torsion and tension tests of the Ti-6Al-4V alloy.
\end{abstract}

Keywords: Garofalo's equation. Creep prediction. Creep deformation. Statistical analysis.

\section{INTRODUCCIÓN}

Se ha probado que la ecuación de Garofalo es de gran interés para el estudio de las propiedades físicas de los materiales metálicos policristalinos (1-4). Asimismo, su importancia para el estudio de aspectos tecnológicos del conformado de estos materiales es clave, dado que permite relacionar variables de interés en los procesos de fabricación, como son la tensión de trabajo, $\sigma$, la velocidad de deformación, $\dot{\varepsilon}$, y la temperatura, $T$. El simple ajuste de una ecuación a los resultados experimentales de los ensayos de los materiales no es garantía suficiente de que ésta sea una ecuación de estado, tampoco lo es de que dicha ecuación represente adecuadamente valores experimentales no pertenecientes a la base de

(•) Trabajo recibido el día 12 de septiembre de 1997 y aceptado en su forma final el 9 de marzo de 1998.

(*) Centro Nacional de Investigaciones Metalúrgicas, CENIM (CSIC). Avda. de Gregorio del Amo, 8. 28040-Madrid (España). datos de los ensayos, y mucho menos es garantía de que a partir de la misma se puedan predecir valores de las variables de trabajo que se encuentren alejados o simplemente fuera de los rangos de trabajo de las mismas variables (1-4). Es amplia la relación entre la información física y tecnológica que se puede establecer a partir de una adecuada ecuación de estado para la fluencia plástica estacionaria de materiales metálicos policristalinos. Se puede obtener información sobre la energía de activación del proceso dominante de la fluencia y sobre el tipo de mecanismo microscópico subyacente al proceso macroscópico. Igualmente, se obtiene información sobre volúmenes de activación aparentes de los procesos $(1-4)$.

Está probado (3) que a partir de la ecuación de Garofalo cabe la posibilidad de establecer mapas de estabilidad en el proceso de conformado de los materiales metálicos policristalinos (3). Además, mediante las técnicas numéricas y estadísticas que se han desarrollado, se pueden caracterizar los ensayos en el sentido de "buen o mal 
condicionamiento" de los datos experimentales. Asimismo, es posible analizar si el mal condicionamiento, en su caso, se debe a la presencia de datos considerados como outliers (esto es, puntos de comportamiento anómalo con respecto a la función objetivo) o bien a la presencia de mecanismos competitivos que actúen en paralelo (es sabido que los mecanismos responsables de la fluencia pueden actuar en serie o en paralelo), o bien que presenten zonas de actuación en paralelo con otras en las que predomine uno u otro de los mecanismos. Es posible precisar todos estos detalles usando un método integrado (1-4) que se basa en el acoplamiento de tres métodos encadenados de ajuste progresivo con información que realimenta el proceso, la base de datos y el resultado del ajuste. Se comienza con una linealización de la ecuación de Garofalo:

$$
\dot{\varepsilon}=A \cdot e^{-\frac{Q}{R \cdot T}} \cdot \operatorname{sen} h(\alpha \cdot \sigma)^{\mathrm{n}}
$$

en la forma:

$$
\ln (\dot{\varepsilon})=\ln (A)-\frac{Q}{R \cdot T}+n \cdot \ln (\operatorname{sen} h(\alpha \cdot \sigma))
$$

en la que la variable de linealización, $\alpha$, está sometida a las exigencias necesarias: a) Su recorrido debe incluir todos los valores que verifiquen que $\alpha \cdot \sigma \varepsilon(0,6-1,8)$, según se justifica en (3); b) Los valores de $\alpha$ deben garantizar que se considera cualquier valor de $\sigma$ físicamente compatible con el proceso de trabajo. No obstante, se pueden tomar valores de $\alpha$ entre $10^{-5}$ y 500 (por ejemplo, en una determinada escala) sin que se presente ningún problema (para $\sigma$ en $\mathrm{MPa}$ ).

Con estos controles y otros de discretización adecuados, y con el auxilio de unos parámetros estadísticos que permitan valorar el momento de obtención de un óptimo aproximado ( $F$-de Snedecor, análisis de la varianza, análisis de los residuos, contrastes de hipótesis sobre los parámetros del ajuste, etc.) (1-4), se consiguen los valores iniciales de $\{Q, n, A, \alpha\}$, adecuados para el tratamiento de la base de datos con un método no lineal directo, basado en el de Gauss-Newton, pero adaptado a las necesidades del tipo de función objetivo a tratar, y sobre todo, con un tratamiento de la búsqueda univariada del mejor tamaño del paso de descenso al óptimo, en la dirección de mayor disminución del error, que le hace especialmente apropiado para hallar la solución óptima. Se puede garantizar que, en caso de existencia, se alcanza un óptimo global para el problema planteado. Mejor dicho, se garantiza la obtención de la superficie óptima de ajuste de la ecuación de Garofalo a los datos experimentales.
Estos métodos no lineales están limitados por los controles de convergencia, que son de dos clases: De velocidad y de precisión. Por ello, se precisa un ajuste fino del óptimo global conseguido que se realiza en una etapa final, a la que se da menor significado estadístico, que consiste en iterar por caminos en cortes sobre la superficie mediante líneas a $Q=$ cte., o a $\alpha=$ cte., hasta conseguir una consistencia en los estadísticos y una constancia en los valores de los parámetros que no son mejorados por sucesivos pasos. Esta etapa final no es, estrictamente hablando, un método iterativo tradicional, ya que se controlan los caminos y, a su vez, los intervalos de recorrido de las variables que se han hecho constantes, a diferencia de los métodos iterativos tradicionales que trabajan en lazos cerrados similares a los seguidos por los métodos de punto fijo en la resolución de ecuaciones no lineales. Tradicionalmente, los métodos de resolución de la ecuación de Garofalo, han sido:

- Adopción de valores de los parámetros del ajuste tomados de la bibliografía o de la experiencia, en virtud de la pertenencia del material a alguna familia de materiales con parámetros que se suponían suficientemente conocidos (5).

- Métodos iterativos tradicionales, a partir de ecuaciones algebraicas y que, mediante la relajación, alcanzaban una modificación de los parámetros y una readaptación de la base de datos experimental (6).

- Métodos iterativos por líneas que reducían los ajustes de $\Re^{4}$ a $\Re^{3}$, siendo $\Re$ el espacio de los números reales.

- Métodos no lineales tradicionales, con aportación de valores iniciales próximos a la solución.

Generalmente, los métodos no lineales usados se implementan en algoritmos comerciales proporcionados por paquetes estadísticos o numéricos existentes en el mercado, y los métodos basados en la eliminación de parámetros se desarrollan con herramientas matemáticas sencillas.

Los problemas básicos de la investigación de la validez de la ecuación de Garofalo como ecuación de estado para la fluencia plástica estacionaria para materiales metálicos policristalinos, son:

- Que es una ecuación fuertemente no lineal.

- Que trabaja para el ajuste en $\Re^{4}$.

- Que la función dé error.

$$
E(A, Q, n, \alpha)^{2}=\sum_{\mathrm{i}=1}^{\mathrm{i}=\mathrm{N}}\left(\dot{\varepsilon}_{\mathrm{i}}^{\text {exper. }}-A \cdot e^{-\frac{Q}{\mathrm{R} \cdot \mathrm{T}_{\mathrm{i}}}} \cdot \operatorname{sen} h\left(\alpha \cdot \sigma_{\mathrm{i}}\right)^{\mathrm{n}}\right)^{2}
$$

puede presentar varios óptimos locales, fruto de errores espurios, de menor importancia que un óptimo global, pero en los que el ajuste se puede 
detener dependiendo de los diferentes algoritmos, controles y pasos de la red correspondiente. Por lo tanto, se trata de superficies no suaves de las que se han verificado con diferentes ensayos experimentales los múltiples problemas que presentan.

- Que los óptimos no globales son muy sensibles a aquellos valores del argumento de la función $\operatorname{sen} h(X)$, en los que ésta no es aproximable por exponenciales o potenciales.

- Que la función senh $(X)$ puede tomar tanto valores negativos como positivos, siendo los negativos valores sin sentido físico, y una incorrecta elección de caminos puede llevar para valores de $X$ pequeños a zonas de oscilación entre valores positivos y negativos proporcionando, como se ha comprobado (1-4), resultados inadecuados.

- Que para valores altos de $X$, el error acumulado en la convergencia es muy alto, debido al comportamiento de la función $\operatorname{sen} h(X)$, y de ello se deducen valores cuyos resultados están afectados por grandes errores, tanto mayores cuanto mayor sea el valor de $n$.

Cuando se trata de trabajar con una función o una ecuación de validez ampliamente probada, cuya base física está suficientemente contrastada, la aplicación de esta función a una base de datos experimental se puede realizar con herramientas normalizadas o comerciales, ya que las anomalías no se atribuyen a la ecuación a su forma o dependencia paramétrica, pero cuando precisamente se está investigando sobre la base física, la validez de la forma o la dependencia de los parámetros de la ecuación, no parece lo más adecuado utilizar herramientas que, respecto al científico, se comporten como cajas negras.

Si se utilizan algunos de los programas caracterizados por ser paquetes comerciales del ámbito estadístico o numérico para realizar el ajuste, primero previamente y después posteriormente al desarrollo de este modelo, a pesar de la excelente calidad de los programas suele ocurrir, dadas las singularidades de la ecuación de Garofalo, que:

- Todos los métodos numéricos no lineales comercializados precisan valores iniciales de los parámetros e intervalos de trabajo de los mismos.

- No todos los programas comerciales permiten imponer las restricciones necesarias a la evolución del ajuste.

- Se ha constatado que cuando los valores iniciales se separan un cierto porcentaje de la solución (previamente conocida y utilizada para generar el problema) (1-4), el ajuste diverge o proporciona resultados espurios.

- En algún caso se ha constatado que separaciones de los valores iniciales superiores al $15 \%$ del valor del resultado correcto (para algunos de los parámetros) conducen a error u overflow. Esto quiere decir que en algunos casos se precisan aproximaciones menores del $15 \%$, con intervalos de confianza del ajuste de los parámetros superiores al $15 \%$ en el error (1-4).

- Para quien conoce los métodos numéricos, es sabido que los métodos no lineales multivariables de uno u otro tipo descansan, en la mayoría de los casos, en búsquedas locales univaluadas, en la selección del camino y tamaño de paso hacia la convergencia, y se ha constatado que los programas comerciales no permiten acceder al nivel de las subrutinas de los ajustes univaluados. Esto es, se puede elegir, en muchos casos, entre diversos métodos generales: Newton, Gauss-Newton, Simplex, método de pérdida, métodos de pasos descendentes, cruzados, de gradientes conjugados, de máxima-verosimilitud, etc. Pero en ningún caso se ha encontrado que se pueda acceder a modificar las características de las rutinas en la búsqueda univaluada cuando es necesaria.

Por otra parte, se ha constatado que diferencias en la tercera cifra decimal, en algunas de las variables de trabajo como es el caso de la velocidad de deformación en $s^{-1}$, producen variaciones en los resultados de los parámetros del ajuste. Esto quiere decir que el problema es muy sensible a los errores numéricos del tratamiento. Ningún programa comercial permite modificar operaciones básicas elementales, la prioridad del cálculo; en definitiva, la adecuación absoluta al problema que se está investigando. En el presente trabajo se utiliza un método adecuado al problema, acorde con él, sujeto a sus limitaciones llegando al límite del error numérico del soporte informático. No se precisan valores iniciales. La convergencia y los algoritmos se controlan desde el nivel superior al de los desarrollos en serie, y hasta se controlan las secuencias de cálculo elemental, optimizando el mismo. Por otra parte, se obtiene una información estadística adecuada al problema y no habitual o normalizada, pudiendo discriminar entre el tipo de problema que sufre un ajuste mal condicionado e informar sobre la calidad del mismo y el origen del problema.

Toda la problemática anteriormente expuesta es de por sí compleja, y se basa en que el trabajo es de investigación sobre la validez, e idoneidad de la ecuación de Garofalo, no dando nada por supuesto. Con toda esta problemática se podría suponer que el problema es academicista y que en el marco de la investigación y el desarrollo, el problema no aporta información a un fabricante que tiene que modificar las variables de trabajo cuando se modifican algunas de las solicitaciones. Precisamente, ese ha sido el objetivo último del trabajo. En el marco de este 
modelo y métodos se puede garantizar una capacidad predictiva.

Uno de los aspectos que más pueden ayudar a clarificar la magnitud del problema es el siguiente: existen teorías globales completas cerradas para una matemática numérica de lo lineal. No existe una teoría general de lo no lineal en ningún ámbito de la matemática, entiéndase ecuaciones diferenciales, álgebra, estadística, etc. Existen problemas no lineales concretos, soluciones concretas a problemas no lineales concretos. Pues bien, desde este enfoque se ha trabajado, desarrollando una solución concreta, completa, para un problema no lineal concreto, resolviendo dos aspectos simultáneamente, el matemático y el físico, ya que avanzar en aspectos parciales por uno de los dos caminos, cuando el problema es de esta magnitud, puede conducir a conclusiones erróneas en una u otra forma. Esto es, tomar aproximaciones de base física para facilitar la matemática, o sacar conclusiones físicas a partir de herramientas matemáticas cuyas limitaciones se desconocen, puede introducir errores de difícil control.

La palabra predicción, en el caso de la estadística del ajuste de datos experimentales a funciones objetivos, se usa en una acepción diferente a la que se usa en otros campos de la física. El valor de predicción de una variable dependiente, $y_{\mathrm{i}}^{*}$, es el obtenido mediante la función objetivo del ajuste cuando en lugar de la variable independiente se coloca un valor del recorrido experimental. Sin embargo, la acepción de predicción que interesa en el marco del desarrollo tecnológico es más bien aquella que se usa en meteorología; se busca un valor de la variable dependiente producido a través de la función objetivo del ajuste, a partir de valores de la variable independiente que no pertenezcan al conjunto de datos del ensayo experimental: si pertenecen a los intervalos $\left[x_{\min }, x_{\max }\right]$ será una predicción de interpolación, y si el valor de la variable independiente queda fuera del recorrido experimental será una predicción de extrapolación o, en su sentido más genérico, una predicción. Teniendo buen cuidado de distinguir estas acepciones, el objetivo de este trabajo es validar la capacidad predictiva.

Generalmente, en la bibliografía se observan representaciones gráficas que validan la obtención de unos parámetros u otros a partir de uno o dos puntos de verificación. Se ha decidido realizar el presente trabajo a partir de datos experimentales de amplios recorridos de las variables, obtenidos por diferentes métodos experimentales, y verificando la capacidad de predicción no sobre uno o dos puntos sino sobre un $25 \%$ de los datos. Este valor porcentual de los datos experimentales utilizados para calibrar la capacidad predictiva no se ha usado para el tratamiento numérico ni ha sido seleccionado con otro criterio que el de quedar lo más alejado posible del núcleo de los recorridos de las variables experimentales.

Se ha trabajado con diferentes materiales y en distintas condiciones, a partir de ensayos experimentales diferentes, obteniéndose una amplia serie de resultados de gran interés.

La aparición en este ámbito de la ecuación de Garofalo se produce cuando los investigadores van aumentando los recorridos en tensiones de trabajo, velocidades de deformación y temperaturas, y cuando observan que las técnicas de reducción y los tratamientos matemáticos tradicionales conducen a problemas insolubles (7-11). Se postula la ecuación de Garofalo, en principio heurísticamente, después fenomenológicamente (12) y cada día más con soporte de modelos físicos (3), para resolver los problemas que plantean los resultados experimentales para amplios recorridos de las variables. Al mismo tiempo, se consigue información física de la misma que permite interpretar los resultados y obtener conclusiones sobre la física microscópica del proceso de deformación. Su validación va permitiendo obtener resultados sobre la física y la tecnología del problema, que antes se obtenían para intervalos reducidos a partir de las leyes potenciales y de las leyes exponenciales, por separado.

En este trabajo se ha abordado la cuestión avanzando en el conocimiento del problema matemático y del problema físico en cooperación, que es la forma óptima para no obtener conclusiones precipitadas y erróneas.

\section{MATERIALES Y MÉTODO EXPERIMENTAL}

El presente trabajo se ha realizado con un material cuya importancia en diferentes áreas tecnológicas le ha convertido en objeto de múltiples estudios, la aleación Ti-6Al-4V, que en las condiciones precisas tiene un comportamiento superplástico $(n=2)$, se ha sometido a ensayos de tracción para bajas velocidades de deformación donde el mecanismo de fluencia predominante será el de deslizamiento de fronteras de grano, si su tamaño es suficientemente pequeño. Para velocidades elevadas se ha utilizado un banco de torsión, ya descrito en otras publicaciones (3). Los datos de ambos ensayos se han convertido en tensiones eficaces y deformaciones eficaces según el criterio de Von Mises (13-16) de forma que sean coherentes. En suma, se ha partido de un material de grano fino que se ha sometido a deformaciones comprendidas entre $7 \cdot 10^{-5}$ y $5 \mathrm{~s}^{-1}$, en el rango de temperaturas $1.073-1.270 \mathrm{~K}$ que garantizan una cantidad suficiente de estructura $\alpha+\beta$ utilizada habitualmente en procesos de conformado.

La composición química de la aleación Ti-6Al$4 \mathrm{~V}$, se muestra en la tabla I. 
TABLA I.- Composición química del Ti-6Al-4V, en $\%$ en masa

TABLE I.- Chemical composition of Ti-6Al-4V, $\%$ in mass

\begin{tabular}{|c|c|c|c|c|c|}
\hline $\mathrm{Al}$ & $\mathrm{V}$ & $\mathrm{Fe}$ & $\mathrm{O}$ & $\mathrm{N}$ & $\mathrm{Ti}$ \\
\hline 6,34 & 3,92 & 0,15 & 0,17 & 0,02 & Bal. \\
\hline
\end{tabular}

Se han realizado ensayos mediante torsión y tracción, con probetas cuya estructura era de grano fino $(7-19 \mu \mathrm{m})$. Los ensayos de torsión se han llevado a cabo en una máquina que ya se describió en (3). Los ensayos de torsión y tracción se realizaron mediante ensayos de variación de velocidad controlados por ordenador, cubriendo varios órdenes de magnitud en velocidades de deformación. Se dispone de variaciones experimentales en velocidades de deformación comprendidas entre $7 \cdot 10^{-5} \mathrm{~s}^{-1}$ y $5 \mathrm{~s}^{-1}$. El ensayo de torsión cubre desde $7 \cdot 10^{-2}$ a $5 \mathrm{~s}^{-1}$, y el de tracción desde $7 \cdot 10^{-5}$ a $7 \cdot 10^{-3} \mathrm{~s}^{-1}$. El intervalo de temperaturas fue de 1.073-1.270 K. En este rango, una fase dúplex $(\alpha+\beta)$ es la estructura estable de la aleación. Todos los ensayos se han realizado bajo atmósfera de helio para minimizar diversos efectos. Los datos de torsión (par aplicado y vueltas por segundo) se han reducido mediante las conversiones adecuadas (13-16). Dichos datos se ofrecen en la tabla II.

\section{RESULTADOS Y DISCUSIÓN}

Las características mecánicas de la aleación estudiada son suficientemente conocidas y no son el objeto estricto de este trabajo. Deben servir de punto de partida para una evaluación de las mismas o bien de las características de la instalación industrial de conformado.

En resumen, se tienen varios tipos de resultados: los de los ensayos de simulación que se muestran en la tabla II -de los cuales se ha utilizado sólo la primera parte como base de datos para obtener la función predictiva- y los puntos extremos para comprobar su eficiencia.

La representación de los resultados experimentales se muestra en las figuras 1 y 2 . Se han seleccionado los valores más extremos de los ensayos para verificar la capacidad predictiva. También se han seleccionado valores de velocidad de deformación por encima del valor máximo y por debajo del valor mínimo, que se han utilizado para el tratamiento numérico. En la zona de bajas velocidades de deformación se han tomado valores para verificar la predicción de hasta un orden de magnitud más bajos que los utilizados para el tratamiento matemático, y en la zona de altas velocidades de deformación se
TABLA II.- Resultados de los ensayos experimentales de torsión y de tracción

TABLE II.- Experimental data from torsion and tensile tests

\begin{tabular}{|c|l|l|c|l|}
\hline \multicolumn{5}{|c|}{ Datos tratados numéricamente } \\
\hline Orden & $T, \mathrm{~K}$ & $\dot{\varepsilon}, \mathrm{s}^{-1}$ & $\sigma, \mathrm{MPa}$ & Ensayo \\
\hline 1 & 1.200 & 0,07 & 72,3 & Tor. \\
2 & 1.200 & 0,2 & 91 & Tor. \\
3 & 1.220 & 0,07 & 63,9 & Tor. \\
4 & 1.230 & 0,2 & 67,4 & Tor. \\
5 & 1.230 & 0,5 & 95,4 & Tor. \\
6 & 1.250 & 0,5 & 68,6 & Tor. \\
7 & 1.250 & 2 & 104 & Tor. \\
8 & 1.243 & 0,07 & 39 & Tor. \\
9 & 1.270 & 0,2 & 51,5 & Tor. \\
10 & 1.270 & 0,5 & 67,6 & Tor. \\
11 & 1.179 & 0,007 & 35,44 & Tra. \\
12 & 1.179 & 0,003 & 25,34 & Tra. \\
13 & 1.179 & 0,001 & 15,78 & Tra. \\
14 & 1.179 & 0,0005 & 9,59 & Tra. \\
15 & 1.173 & 0,007 & 52,59 & Tra. \\
16 & 1.173 & 0,003 & 34,28 & Tra. \\
17 & 1.173 & 0,001 & 21,41 & Tra. \\
18 & 1.123 & 0,007 & 80,23 & Tra. \\
19 & 1.123 & 0,003 & 56,37 & Tra. \\
20 & 1.123 & 0,001 & 35,76 & Tra. \\
21 & 1.123 & 0,0005 & 23,08 & Tra. \\
22 & 1.073 & 0,001 & 109,12 & Tra. \\
23 & 1.073 & 0,0005 & 73,75 & Tra. \\
24 & 1.073 & 0,0005 & 15,33 & Tra. \\
\hline \multicolumn{5}{|c|}{ Datos usados para la predicción } \\
\hline 25 & 1.200 & 2 & 169 & Tor. \\
26 & 1.230 & 1 & 1.124 & Tor. \\
27 & 1.230 & 2 & 1.313 & Tor. \\
28 & 1.250 & 5 & 130 & Tor. \\
29 & 1.173 & 2 & 838 & Tra. \\
30 & 1.123 & 2 & 1.282 & Tra. \\
31 & 1.073 & 3 & 14.769 & Tra. \\
32 & 1.073 & 2 & 4.392 & Tra. \\
33 & 1.073 & 7 & 2.391 & Tra. \\
\hline \multicolumn{5}{|c|}{} \\
\hline \multicolumn{5}{|c|}{} \\
\hline 5
\end{tabular}

han seleccionado datos para la predicción suficientemente alejados del máximo valor empleado en el ajuste. Con respecto a las tensiones de trabajo, las distancias en magnitud son más pequeñas, lo cual es lógico debido a que los recorridos en tensiones son de sólo dos órdenes de magnitud.

El titanio es un material con un valor elevado de energía de falta de apilamiento, $300 \mathrm{MJm}^{-2}$, por lo que la restauración dinámica se puede producir en la fase $\alpha$ (hcp), suponiéndose también valores elevados de falta de energía de apilamiento para la fase $\beta$ (bcc); es sencillo que se produzcan en esta fase procesos de deslizamiento cruzado y trepado 


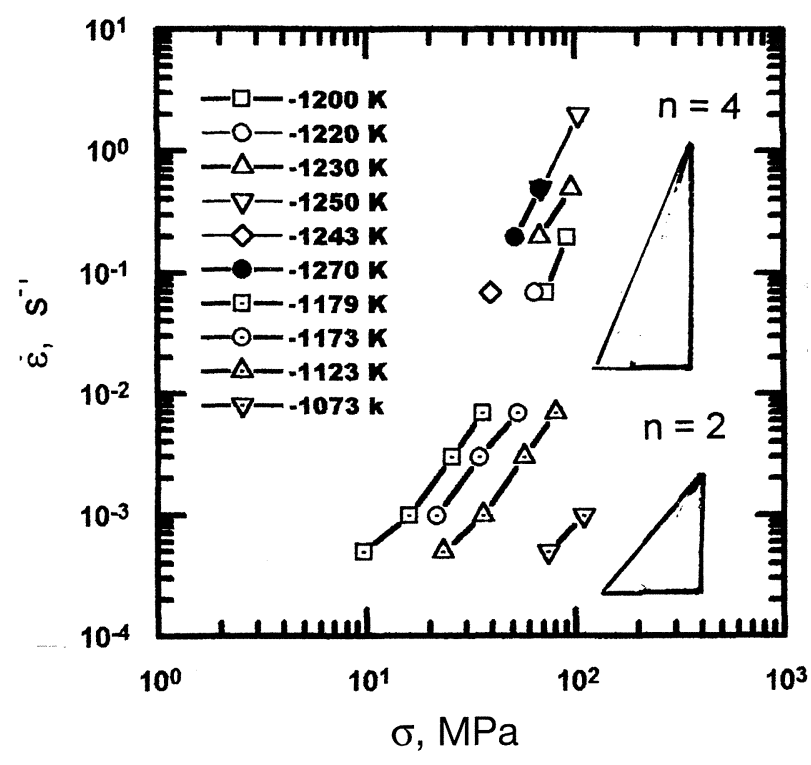

Fig. 1.- Representación de los datos de ensayos experimentales correspondientes a la tabla II (primeros 24 datos).

FIG. 1.- Representation of experimental values of table II (the first 24 values).

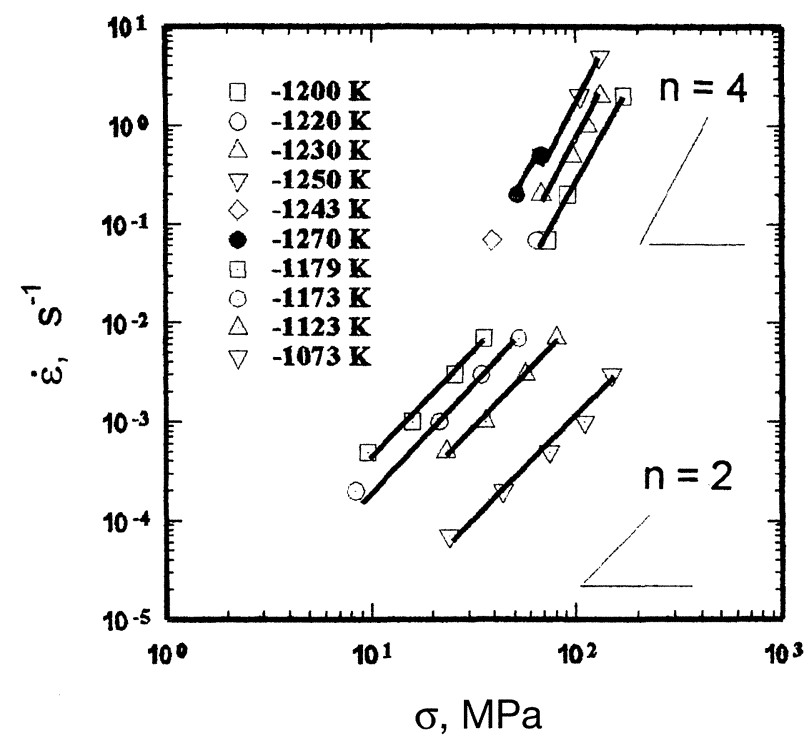

FIG. 2.- Representación de los datos de ensayos experimentales correspondientes a la tabla II (completa).

FIG. 2.- Representation of experimental values table II (all the values).

(17-19), pero si la velocidad de enfriamiento es suficientemente rápida, la fase $\beta$ sufre una transformación martensítica que elimina cualquier evidencia de subestructuras de deformación, siendo por ello difícil de probar una restauración dinámica. La aleación Ti-6Al-4V permite una estructura de dos fases por debajo de la temperatura de transición
(17-19). Las energías de activación, para la autodifusión en titanio puro para las fases $\alpha$ y $\beta$ son, respectivamente, 169.147 y $152.818 \mathrm{~kJ} \mathrm{~mol}^{-1}$. En ensayos de aleación con coexistencia de las dos fases son de 470 y $572 \mathrm{~kJ} \mathrm{~mol}^{-1}$ (14), valores mucho mayores que los de la energía de autodifusión.

Al tratar los datos en la primera etapa (de obtención de valores iniciales), se ha usado una discretización de $\alpha$, entre $[0,00001,300]$ con $\Delta \alpha=1$ (teniendo en cuenta que se ha trabajado con $\sigma$ en $\mathrm{MPa}$ (en el caso de querer comparar en $\mathrm{Pa}^{-1}, \alpha \varepsilon$ $\left[10^{-8}, 0,3\right]$ y $\left.\Delta \alpha=0,001\right)$, con lo que se está garantizando la validez del proceso de ajuste en esta etapa (1-4) para valores de $\sigma$ entre $3 \mathrm{~Pa}$ y $10^{5} \mathrm{MPa}$. Los resultados obtenidos son los siguientes: $\{A, Q, n, \alpha\}=$ $\left\{6.938 \cdot 10^{16}, 424,95 \mathrm{~kJ} \mathrm{~mol}^{-1}, 1,99,0,017 \mathrm{~Pa}^{-1}\right\}$. El valor de la varianza de los residuos es de 0,17 . El coeficiente de determinación de Pearson es de 0,983 y $R=0,991$. Luego, el ajuste explica el $98 \%$ de la varianza de los datos. El valor de la función $F$ de Snedecor experimental, resultado del análisis de la varianza, es $F(p-1, N-p, 1-\alpha / 2)=F(2,21,0,975)$, siendo $p=$ número de variables regresoras, $N=$ número de datos, y $\alpha$ el nivel de significación o máximo valor que se está en disposición de admitir en la probabilidad del error de la hipótesis, y que se toma como 0,05 , en cuyo caso $F(2,21,0,975)=$ 607,58 , frente al valor teórico de $F=4,42$, que se puede obtener de cualquier tabla estadística de la función $F$ de Snedecor. Este valor hace altamente significativo el resultado. Esto quiere decir que la función objetivo utilizada, en su forma linealizada, ec. [2], es para el valor $\alpha$ del ajuste de 0,017, altamente adecuada con los valores del ajuste anteriormente mencionados (1-4). Los intervalos de confianza al $95 \%$ de la misma son, para cada uno de los parámetros del ajuste y en las unidades indicadas en su caso, los siguientes: $A \varepsilon\left[4,9 \cdot 10^{15}\right.$, $\left.9.819 \cdot 10^{17}\right] \mathrm{s}^{-1}, Q \varepsilon[398,76,451.135] \mathrm{kJ} \mathrm{mol}^{-1}, n \varepsilon$ $[1.835,2.155]$. Un $100 \%$ de los residuos reducidos se encuentran dentro del intervalo $[-1,96,1,96]$, lo cual quiere decir que tipificada la variable de los residuos, se comporta en primera aproximación como una variable estadística con distribución normal. El valor de $T 11$ (1-4) es de 2,92, suficientemente adecuado como para garantizar que no existe heterocedasticidad de los residuos. La autocorrelación posible de los residuos y la función de densidad de los mismos, junto con el ensayo de normalidad $\chi^{2}$ de Pearson, se ofrecen en las figuras 3, 4 y 5 . Se puede observar que la función de densidad de los residuos es suficientemente normal (esto lo marca el ajuste de la curva de distribución normal, ya que los histogramas son relativos por depender de la amplitud de las bandas), el ensayo $\chi^{2}$, para la normalidad, es adecuado, y la función de autocorrelación presenta una anomalía debida a la 


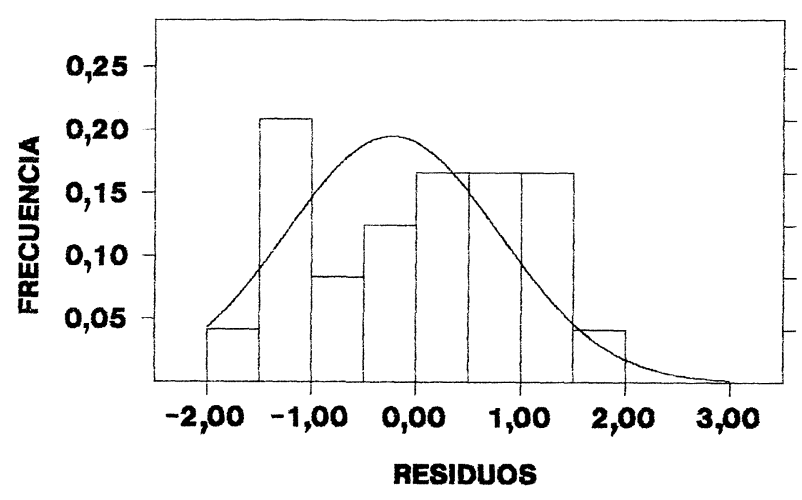

FIG. 3.- Función de densidad de la distribución de los residuos del ajuste.

FIG. 3.-Density function of residuals distribution for the best fitting.

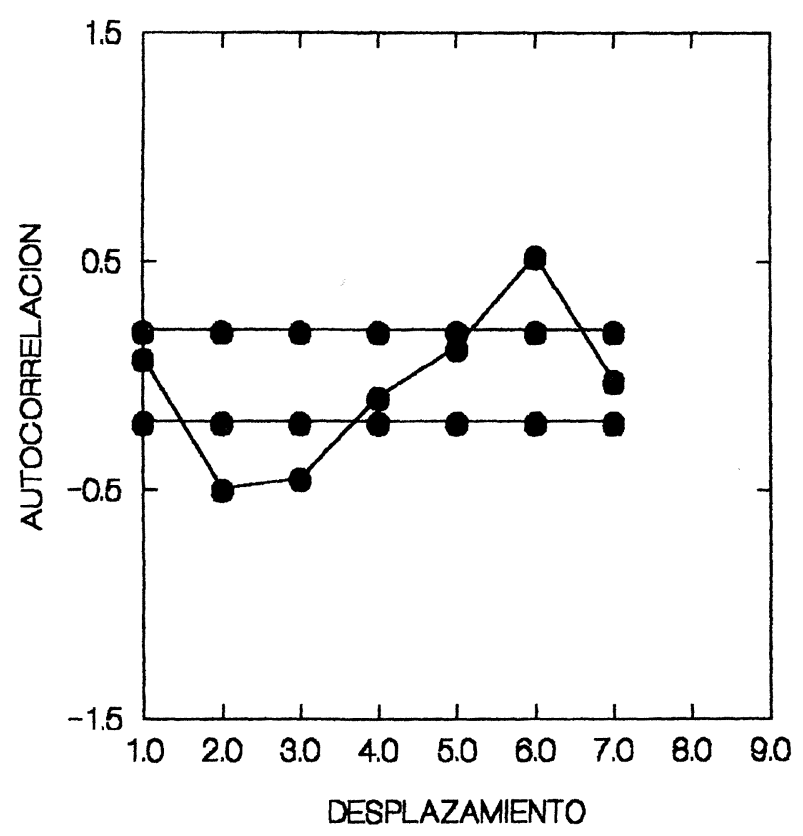

FIG. 4.- Autocorrelación de los residuos del ajuste.

FIG. 4.- Autocorrelation function of residuals for the best fitting.

dependencia que condiciona la elección de la base de datos.

Cuando se utilizan los anteriores valores obtenidos en la primera etapa para ser tratados con el método no lineal directo, se obtiene la siguiente ecuación, fruto del ajuste por el método de GaussNewton modificado (1-4):

$\dot{\varepsilon}=6,9 \cdot 10^{16} \cdot e^{-\frac{425.04 \mathrm{kmmo}^{-1}}{8.3736 \cdot \mathrm{T}}} \cdot \operatorname{senh}(0,0171 \cdot \sigma)^{1.992}$

con un valor de la varianza de los residuos de 0,13 que mejora ligeramente el valor de la etapa anterior.

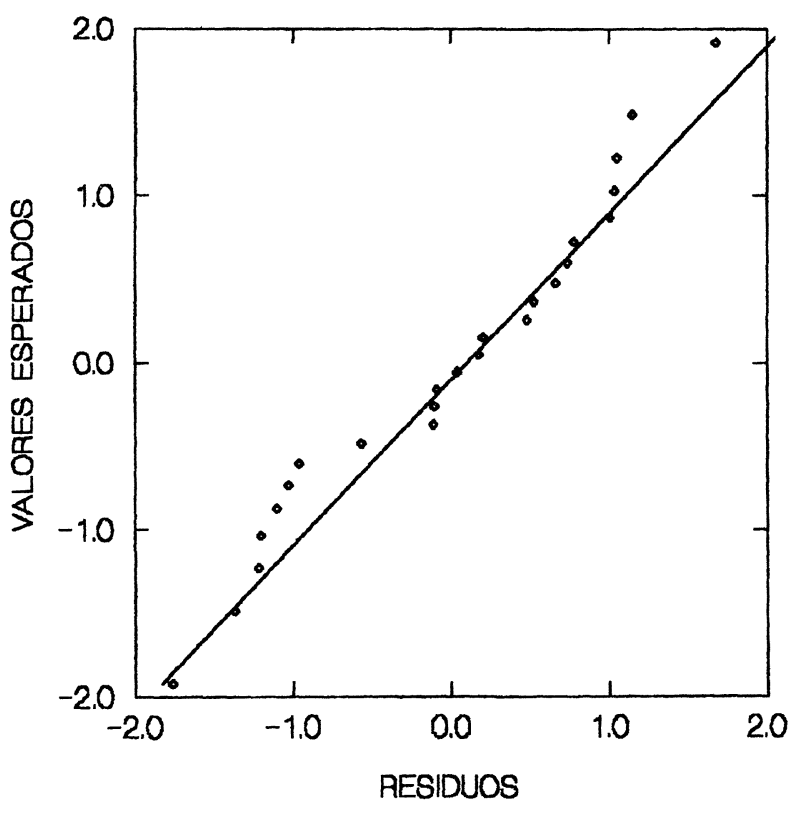

FIG. 5.- Ensayo $\chi^{2}$ para la normalidad de los residuos.

FIG. 5.- Test $\chi^{2}$ for the residuals normality.

Tras la segunda etapa se someten los resultados a un tratamiento por caminos en $\Re^{2}$, por líneas a $Q, \mathrm{y}$ $\alpha$ constantes, que conducen al siguiente resultado:

$\dot{\varepsilon}=8,32 \cdot 10^{16} \cdot e^{-\frac{427.1 \mathrm{kJmol}-1}{8,3736 \cdot \mathrm{T}}} \cdot \operatorname{senh}(0,0172 \cdot \sigma)^{1.987}$

los últimos valores de la iteración para $R$ son $R=$ 0,976 . En este caso, las iteraciones por caminos no parecen mejorar el ajuste de la etapa segunda del programa (esto no es frecuente, pero cuando sucede da una idea de la calidad global de los datos o de la adecuación de la función objetivo).

Por último, y para realizar el análisis estadístico, se han tomado los puntos dato números 25 al 33 de la tabla I y, a partir de los valores de $T$ y $\sigma$, con la ayuda de la ec. [4] se han obtenido unos valores de $\varepsilon_{\text {predicción }}$ que se pueden observar en la tabla III.

Los valores obtenidos son realmente muy adecuados dada la dificultad numérica del problema.

En la figura 6 se pueden observar los errores relativos del ajuste frente a las velocidades de deformación. Los círculos llenos son los errores relativos de los valores de predicción, y los vacíos son los correspondientes a los datos empleados en el ajuste; se observa que para un ajuste como el de la ec. [4], que es un buen ajuste, siete de los nueve datos (un $78 \%$ ) de los empleados para valorar la predicción quedan en la misma banda de error que los 24 datos empleados para llevar a cabo el ajuste.

En la figura 7 se observa una representación similar, en este caso de los errores relativos frente a la tensión de trabajo. La figura 8 muestra la 
TABLA III.- Resultado de los valores predichos por la ec. [4], para los nueve puntos del ensayo experimental no utilizados pra el ajuste

TABLE III.- Results of predicted values from eq. [4] for nine test points not used for the fitting

\begin{tabular}{|c|l|c|}
\hline $\begin{array}{c}\dot{\varepsilon} \text { (medida), } \\
\mathrm{s}^{-1}\end{array}$ & $\begin{array}{c}\dot{\varepsilon} \text { (predicha), } \\
\mathrm{s}^{-1}\end{array}$ & $\begin{array}{c}\text { Error } \\
\text { relativo }\end{array}$ \\
\hline 2 & 2,32 & 0,16 \\
2 & 2,32 & 0,16 \\
1 & 0,91 & 0,09 \\
2 & 1,77 & 0,11 \\
5 & 3,3 & 0,34 \\
0,0002 & 0,00023 & $-0,17$ \\
0,0002 & 0,00008 & 0,60 \\
0,003 & 0,007 & $-1,49$ \\
0,0002 & 0,000134 & 0,33 \\
0,00007 & 0,000035 & 0,5 \\
\hline
\end{tabular}

necesidad del ajuste mediante la ecuación de Garofalo. De tratarse de recorridos cortos de las variables podrían haberse ensayado ajustes potenciales, pero dado que la constante de Zener-Hollomon pre-

$$
Z=\dot{\varepsilon} \cdot e^{-\frac{Q}{R \cdot T}}
$$

senta recorridos de cinco órdenes de magnitud y en tensión de dos órdenes de magnitud, resulta inade-

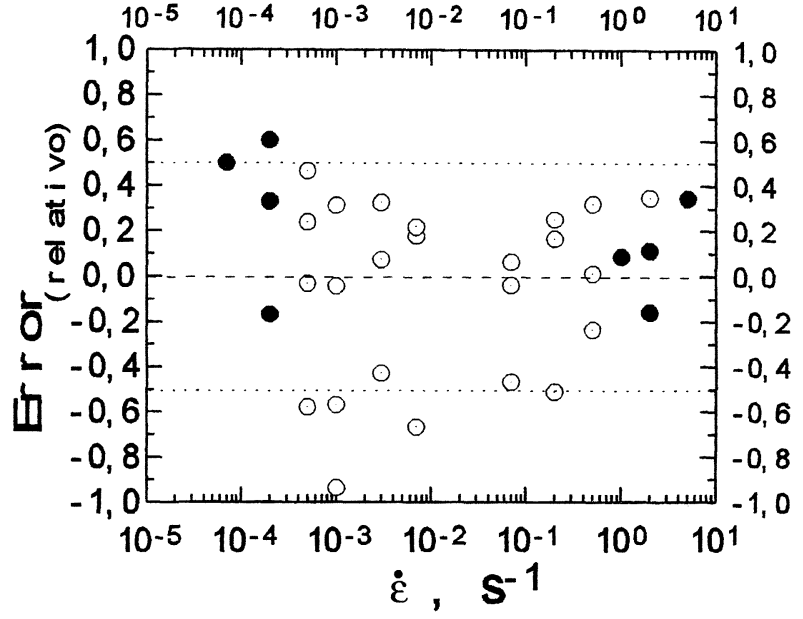

FIG. 6.- Error relativo del ajuste para los puntos de la tabla II frente a la velocidad de deformación (los puntos llenos son los usados para la predicción).

FIG. 6.- Relative error of fitting data of table II, vs strain rate (the black circles were used to forecast).

cuádo cualquier ensayo de ajuste mediante leyes potenciales para todo el recorrido.

En la figura 8 se puede ver la adecuación de los puntos de predicción al ajuste, y se pone de manifiesto que en un recorrido de cinco órdenes de magnitud en $Z$ y de dos órdenes en tensión, los valores predichos quedan perfectamente ajustados a la

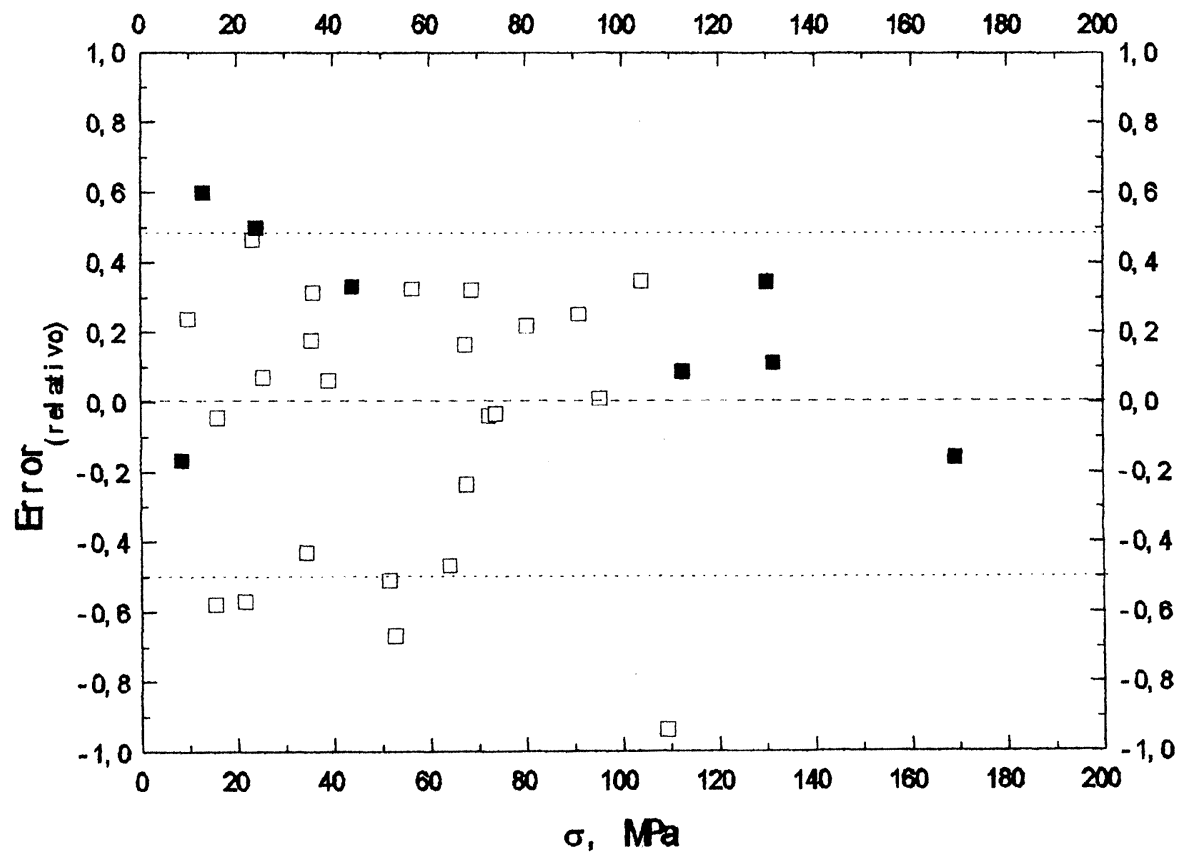

Fig. 7.- Error relativo del ajuste para los puntos de la tabla II frente a la tensión de trabajo en MPa (los círculos negros son los datos usados para la predicción).

FIG. 7.- Relative error of fitting data of table II data vs stress (MPa) (the blacks circles were used to prediction). 


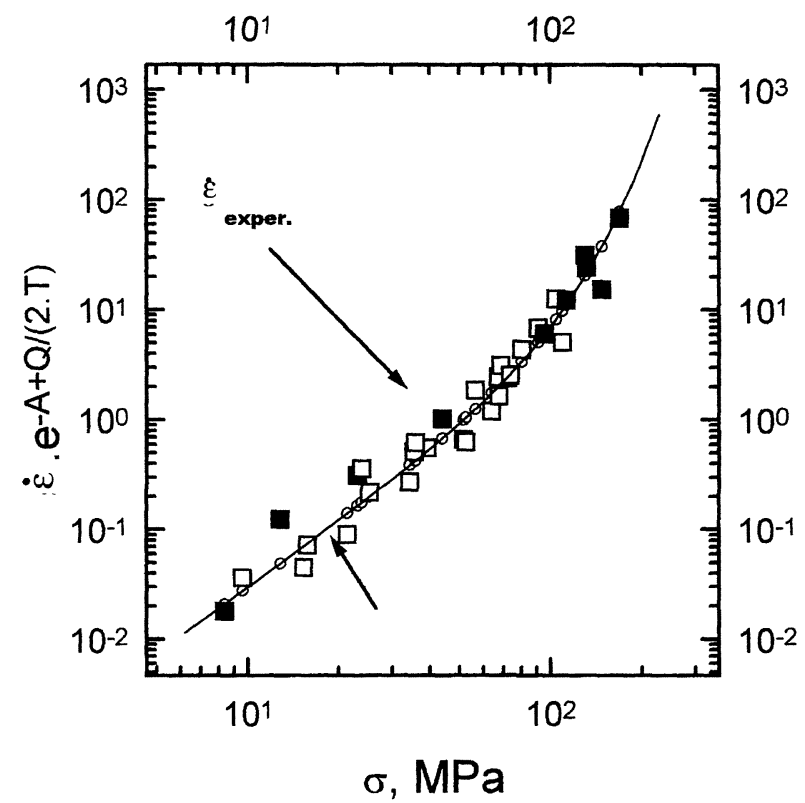

FIG. 8.- Representación del factor $Z$ de ZenerHollomon compensado con la constante $A$ de entropía, frente a la tensión de trabajo $\sigma$ en $\mathrm{MPa}$. Se puede observar la variación continua del posible exponente $n$, de las leyes potenciales, dado que el recorrido es suficientemente amplio.

FIG. 8.- The factor Z (Zener-Hollomon) representation vs stress $\sigma(M P a)$. The continuous variation of exponent $\mathrm{n}$ of the potential laws, can be observed due to the large ranges of variables work.

mejor función obtenida. Se observa que en esta representación, en la que las tres variables se han reducido a dos ejes, aunque los cuadros llenos de los valores de predicción quedan en algunos casos en el centro del recorrido, sólo es un efecto de proyección de la temperatura, ya que son valores extremos en velocidad de deformación y tensión de trabajo.

En la figura 9 se muestra la representación del factor $Z$ de Zener-Hollomon normalizado a la constante $A$, de entropía, frente a la variable $\operatorname{sen} h(\alpha \sigma)$, en cuyo caso la representación, como es sabido, debe ser una recta. Se pueden observar varias cosas en dicha representación. Por una parte, la línea central marcada por puntos pequeños es el ajuste de la ec. [4], usando los valores de $T$ y tensión para generar $\varepsilon$ (teórico), y su representación frente a la función $\operatorname{sen} h(X)$, desarrollan una recta de pendiente $n=$ 1,99. Los círculos abiertos son los valores experimentales de la tabla I que se han ajustado mediante una regresión lineal elemental obteniendo en la forma $y=a+b \cdot x$, para $a=0$ y para $b=1,99$, esto es absolutamente coincidente con el ajuste representado por la ec. [4]. Cuando sucede esto, que no ocurre siempre, el ajuste conseguido mediante el

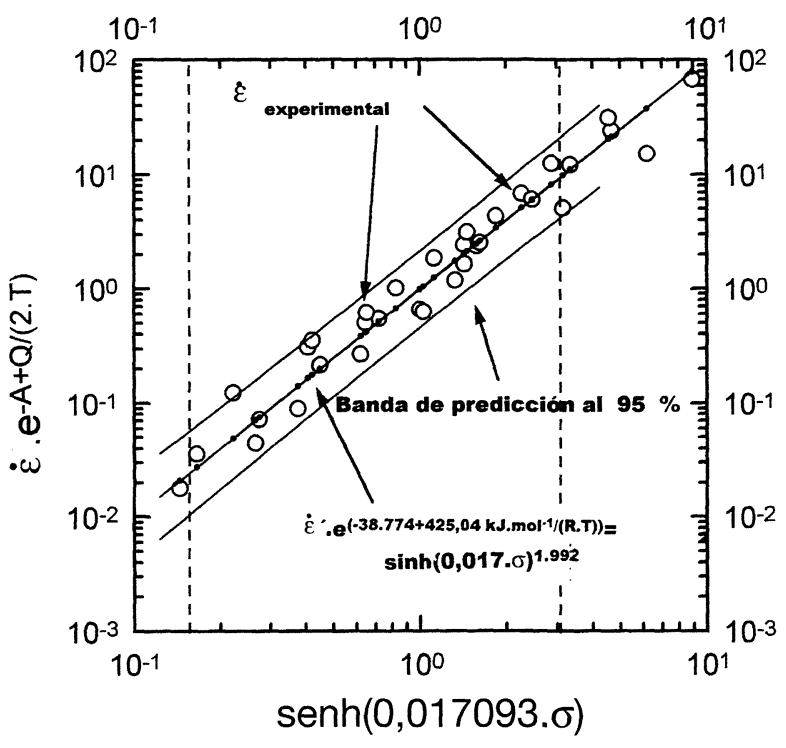

FIG. 9.- Representación de la línea recta del mejor ajuste entre el factor $Z$ de Zener-Hollomon, compensado con la constante $A$ de entropía, frente a $\operatorname{sen} h(\alpha \sigma)$. El ajuste es una recta de pendiente $n=$ 1,99. Los puntos negros corresponden a los valores usados para la predicción. Se señalan las bandas de predicción al $95 \%$ de confianza.

FIG. 9.- Best fitting representation of ZenerHollomon factor (compensate with Entropy constant A) vs senh $(\alpha \sigma)$. The fitting corresponds to a straight line with $\mathrm{n}=1.99$. The blacks dots were used to prediction. Prediction bands for $95 \%$ reliability can be observed.

método que se ha desarrollado es óptimo. Esto se puede deducir sin acudir a este último contraste.

No obstante lo anterior, el ajuste producido por la ec. [4] es algo mejor que el de la recta de regresión de los valores experimentales en la forma representada por la figura 9, ya que en este ajuste se produce un error en los residuos del $15 \%$, mientras que en la ec. [4], sólo se produce del $13 \%$, lo cual es irrelevante en la representación gráfica de la figura 9. También se puede apreciar en la figura 9 que los valores fruto de la predicción que quedan fuera de las líneas verticales discontinuas (intervalo de la región de trabajo en el ajuste) presentan una adecuación a la recta de la ec. [4], inmejorable en algunos casos. Las denominadas bandas de predicción se pueden obtener a partir de los resultados estadísticos del tratamiento de nuestro método y representan unos intervalos de confianza dentro de los cuales se espera, con una confianza del $95 \%$, que se encuentren cualesquiera de los valores predichos a partir de la ec. [4]. Estas bandas se van abriendo tanto más cuanto mayor es la lejanía de la región limitada por las líneas verticales discontinuas $\mathrm{y}$, probablemente a partir de dos ordenes de 
magnitud, su fiabilidad disminuya drásticamente. Los rangos de fiabilidad en los que se puede garantizar la presencia de los valores predichos son suficientes. Cabe destacar también que la amplitud de las bandas de predicción no es pequeña; se puede observar que es de medio orden de magnitud en $Z$ normalizado a $A$, o lo que es lo mismo, el error puede llegar a un cuarto de orden de magnitud.

En la figura 10 se contempla algo sustancialmente diferente: las bandas de intervalo de confianza del ajuste al $95 \%$, que representan una región del plano en la cual se puede encontrar la representación de cualesquiera de los ajustes que se pueden producir con los 24 puntos de los ensayos experimentales de la tabla I. Esto quiere decir que se tiene un $95 \%$ de probabilidad de que la verdadera representación de la mejor ecuación de Garofalo, real pero inasequible con estos datos, se encuentre dentro de esas bandas.

En la figura 11 se han representado las curvas de igual nivel para valores de $\ln (\varepsilon)$ frente a la tensión y a la temperatura; en ella se observan los puntos $A$ y $B$ que corresponden, en el primer caso, a valores experimentales de $\{T, \sigma, \hat{\varepsilon}\}=\{1.250,130,5\}$, en las unidades anteriormente indicadas, para el punto $B$ $\{1.073,23,9,0,00007\}$, siendo los valores de las curvas de nivel correspondientes por interpolación 3,6 y 0,00004 , con los errores de 28 y $43 \%$. En la figura 12 se ha representado con los valores de $\{A, Q, n, \alpha\}$ correspondientes a los del ajuste represen-

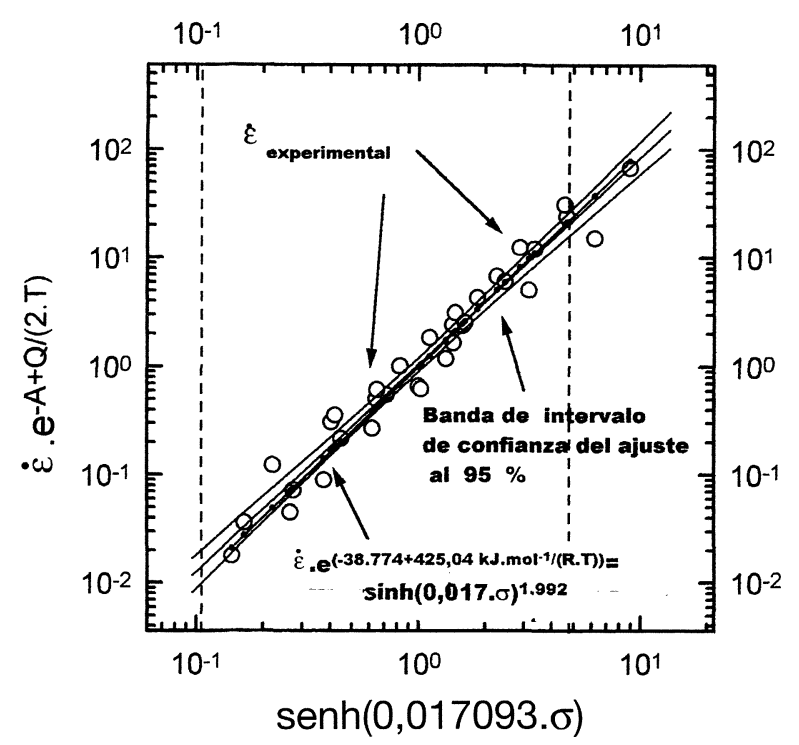

Fig. 10.- Representación del mejor ajuste de la ecuación de Garofalo a los datos de la tabla II, con los intervalos de confianza para el mejor ajuste.

FIG. 10.-Best fitting representation for Garofalo's equation of table II data, with reliability intervals for the best fitting.

tado por la ec. [4]. Esto es, las curvas de igual nivel son las isolíneas de la tensión de trabajo frente a la velocidad de deformación y a la temperatura. En esta figura, los puntos $C$ y $D$ representan los resultados

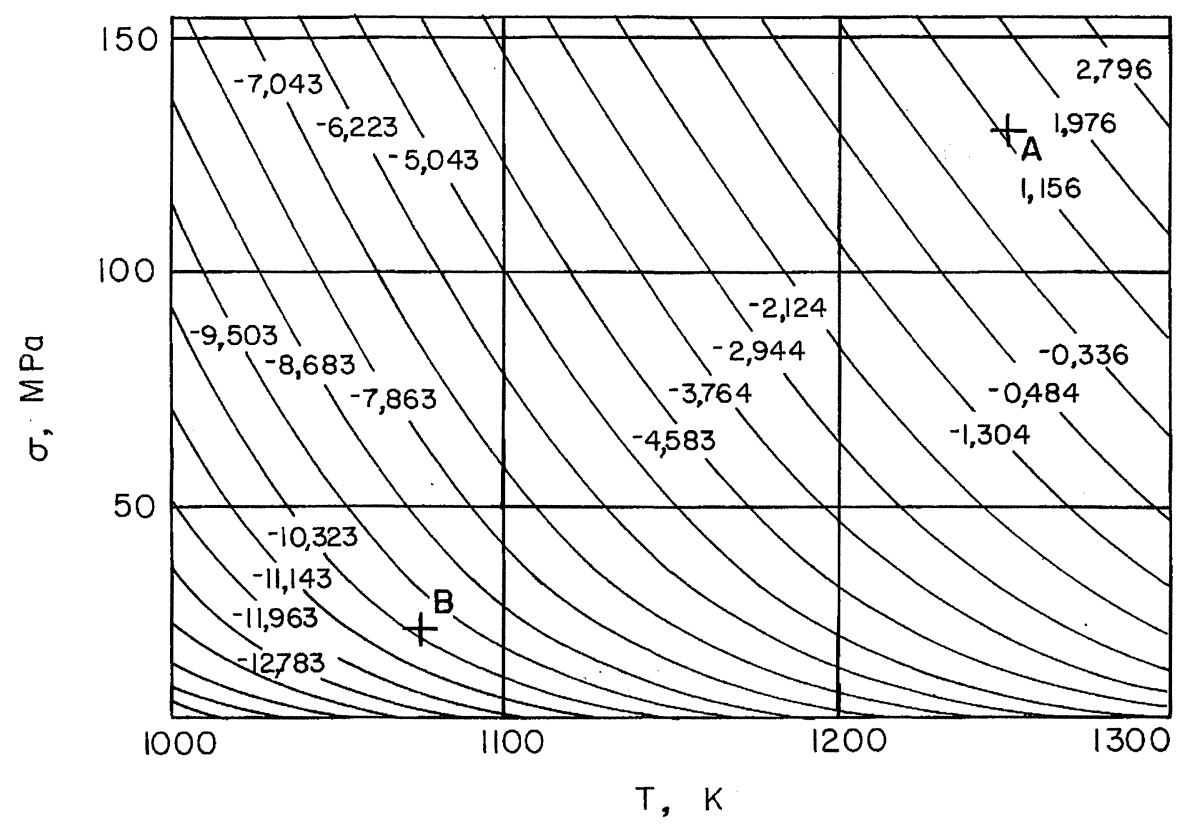

FIG. 11.- Mapa de isolíneas correspondientes a la ec. [4]. Las curvas de igual nivel corresponden a valores iguales de $\ln (\dot{\varepsilon}),\left(\dot{\varepsilon}\right.$ en s$\left.^{-1}\right)$.

FIG. 11.-Isolines for the eq. [4]. The lines of equal level correspond to similar $\ln (\dot{\varepsilon}),\left(\dot{\varepsilon}\right.$ en $\left.s^{-1}\right)$. 


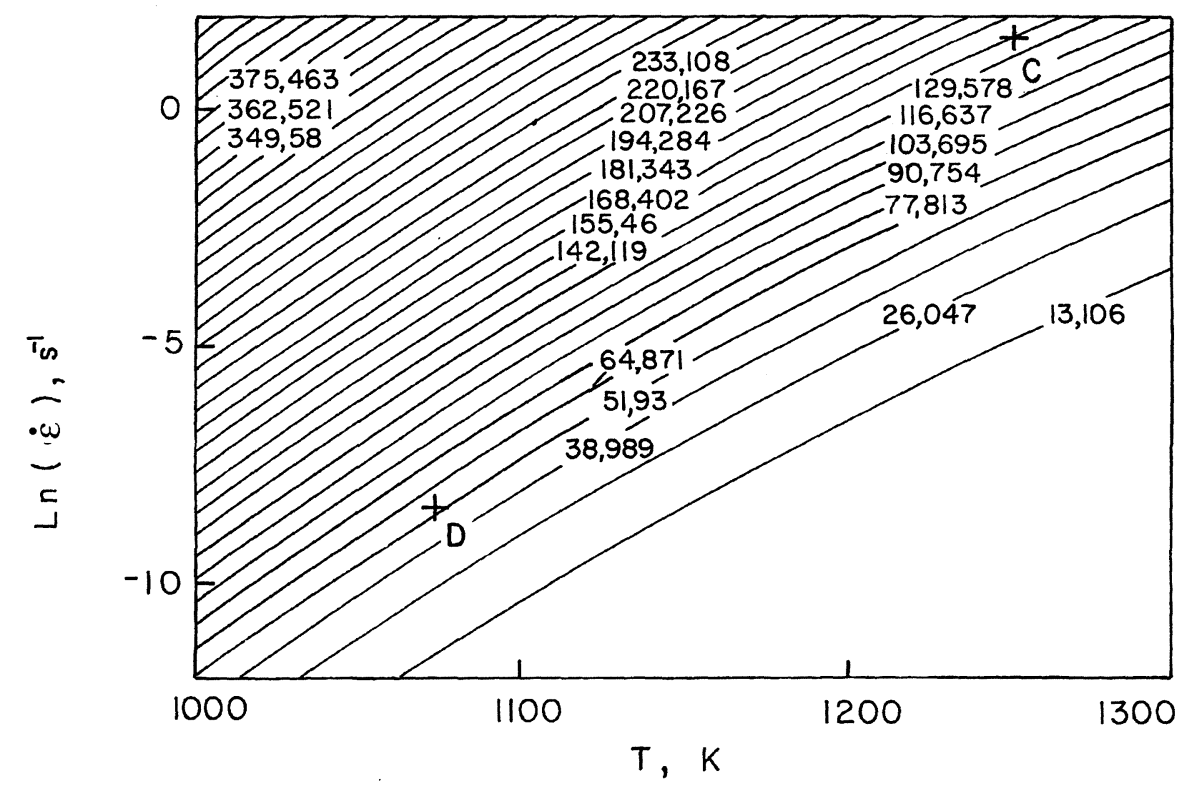

FIG. 12.- Mapa de isolíneas correspondientes a la ec. [6]. Las curvas de igual nivel corresponden a valores iguales de la tensión $\sigma$ en MPa.

FIG. 12.- Isolines for the eq. [6]. The lines of equal level correspond to similar $\sigma$ in $\mathrm{MPa}$.

$$
\sigma=\frac{1}{\alpha} \cdot \operatorname{arcsen} h\left(\left[\frac{\dot{\varepsilon} \cdot e^{\frac{Q}{\mathrm{R} \cdot \mathrm{T}}}}{A}\right]^{\left[\frac{1}{\mathrm{n}}\right]}\right)
$$

experimentales respectivamente para $C\{1.250 \mathrm{~K}, 5$ $\left.\mathrm{s}^{-1}, 30 \mathrm{MPa}\right\}$ y para $D\{1.073,0,0002,43,9\}$, siendo los resultados de las proyecciones (predicciones) de 142 y $52 \mathrm{MPa}$, respectivamente. Por tanto, los errores relativos son el 9 y el $18 \%$. Los errores relativos en la proyección (predicción) de tensiones a partir de velocidades de deformación y de temperaturas son mucho menores que los producidos en las proyecciones de velocidades de deformación a partir de los valores de tensión y de temperatura, y esto se debe a las diferentes características de las funciones inversas que las definen en cada caso (a las variables). La figura 13 muestra la representación tridimensional de la superficie asociada a la ec. [4], de cuyas proyecciones se pueden inferir las predicciones correspondientes, según se elija uno u otro plano de proyección.

Si se realizan las predicciones a partir de la tabla I, pero prediciendo las demandas en tensión a partir de velocidades de deformación y temperaturas, se obtienen los resultados que muestra la tabla IV.

La figura 14 ofrece la representación del error relativo, en valor porcentual, cometido al predecir las tensiones en función de las temperaturas y de las velocidades de deformación, frente a las tensiones experimentales. En la misma representación gráfica se muestra el mejor ajuste lineal entre los errores y

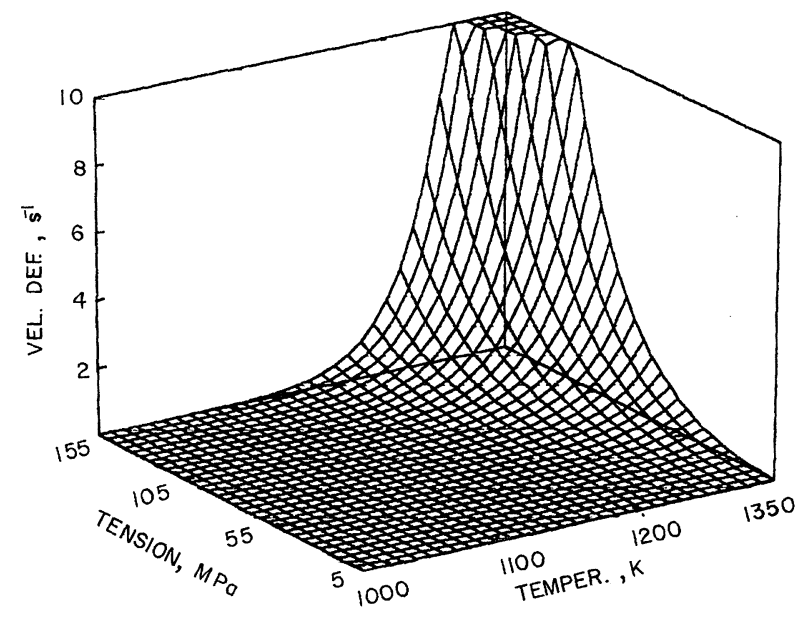

FIG. 13.- Representación de la superficie dada por la ec. [4].

FIG. 13.- Surface representation from eq. [4].

las tensiones experimentales, siendo obvio que hay un cierto sesgo, aunque débil, y siendo la regresión casi despreciable. En cualquier caso, se puede constatar que para tensiones más bajas parece ser mayor el error. En la figura 15 se representan los mismos errores frente a las velocidades de deformación, en escalas logarítmicas, siendo evidente el sesgo de los errores que hace que estos tiendan a aumentar cuando disminuyen las velocidades de deformación. La regresión, en este caso, es de mayor calidad y se establece entre los logaritmos de ambas escalas, por lo que la dependencia es potencial en la forma: 
TABLA IV.- Resultados para las predicciones en tensión de los datos 25 a 33 de la tabla I a partir de la ec. [4]

TABLE IV.- Results of predicted stress from data 25 to 33 of table I using ec. [4]

\begin{tabular}{|c|c|c|c|}
\hline Orden & $\begin{array}{c}\sigma_{\text {Predicción }}, \\
\mathrm{MPa}\end{array}$ & $\begin{array}{c}\sigma_{\text {Experimental }}, \\
\mathrm{MPa}\end{array}$ & $\begin{array}{c}\text { Error relativo, } \\
\%\end{array}$ \\
\hline 25 & 169 & 165 & 2 \\
26 & 115,4 & 112,4 & 3 \\
27 & 135 & 131,3 & 3 \\
28 & 142,6 & 130 & 10 \\
29 & 7,854 & 8,38 & 6 \\
30 & 20,259 & 12,82 & 58 \\
31 & 121,906 & 147,69 & 17 \\
32 & 52,175 & 43,92 & 19 \\
33 & 33,218 & 23,91 & 39 \\
\hline
\end{tabular}

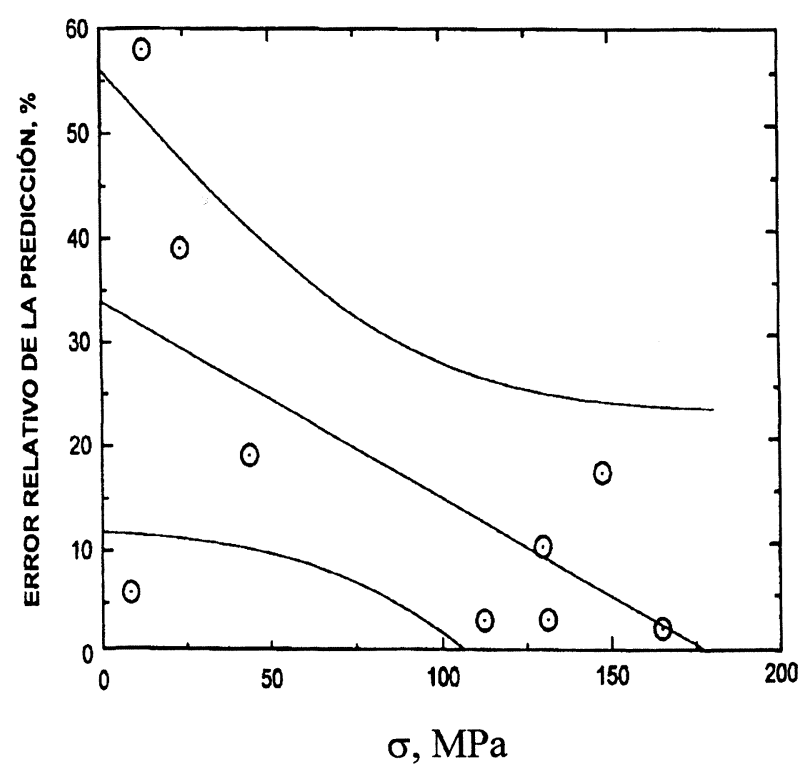

Fig. 14.- Representación de los errores relativos de las predicciones de los casos 25 a 33 de la tabla II, para las tensiones, frente a las tensiones experimentales en MPa. Errores determinados a partir del mejor ajuste de los datos, con los intervalos de confianza del ajuste lineal de los datos.

FIG. 14.- Relative errors representation of predictable stresses of cases 25 to 33 in table II vs experimental stresses (MPa). Errors assessed with reliability intervals of the best lineal data fitting.

$$
\left[E_{\text {rel. }}\right]=\left[4,37 \cdot \varepsilon^{-0,19}\right]
$$

En la figura 2 se puede apreciar que los resultados de los ensayos muestran dos mecanismos claramente diferenciados, a bajas velocidades de deformación, $n=2$, el material se comporta superplásti-

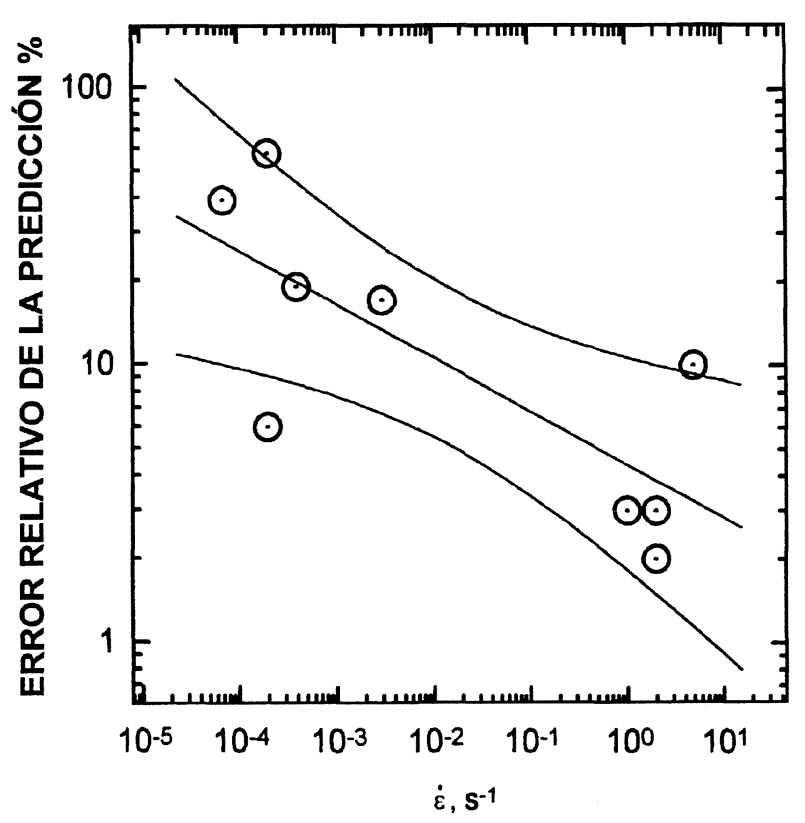

FIG. 15.- Representación de los errores relativos de las predicciones de los casos 25 a 33 de la tabla II, en tensiones frente a las velocidades de deformación de los ensayos, con el mejor ajuste de los datos y los intervalos de confianza del ajuste.

FIG. 15.- Relatives errors representation of predictable stresses of cases 25 to 33 in table II vs experimental deformation rates. Errors assessed with reliability intervals for the best lineal data fitting.

camente (entiéndase que se hace el uso tradicional de interpretación del exponente de las leyes potenciales), siendo el mecanismo de fluencia DBG, mientras que los ensayos cuya velocidad es menor se adaptan a un mecanismo de trepado de dislocaciones, $n=4,5$.

Por otra, parte la solución de la ecuación de Garofalo que se ha obtenido proporciona un valor $n$ $=2$, lo que permite predecir que si las condiciones son adecuadas el material se comporta superplásticamente, ya que en las zonas de muy bajas velocidades $\operatorname{sen}(\alpha \sigma) \approx \alpha \sigma$; por tanto, el valor del exponente $n$ de la ecuación de Garofalo debe coincidir y coincide con el valor de $n$ de las leyes potenciales para DBG si lo hay, además, no contradice el mecanismo de $n=4-4,5$ ya que en esa zona no se puede hacer la sustitución de la función seno-hiperbólico por el arco de la misma.

\section{CONCLUSIONES}

Se ha probado la capacidad de predicción de este método integral de ajuste de la ecuación de Garofalo aplicado a una base de datos, resultado de 
ensayos experimentales de la aleación de Ti-6 Al$4 \mathrm{~V}$, usando 24 puntos para el ajuste y a partir de un ajuste valorado como altamente significativo por la bondad de los parámetros estadísticos se ha procedido a predecir los resultados sobre nueve puntos de ensayo no usados para el ajuste seleccionados por ser los valores extremos de los intervalos de trabajo. La capacidad de predicción se ha mostrado adecuada, tanto en los casos de elevadas como de bajas velocidades, y tanto en los de altas como en los de bajas tensiones, siendo mejor en ambos tipos de predicción (velocidades y tensiones) para valores altos, y siendo mucho mejor el resultado para la predicción en tensiones que en velocidades de deformación.

Se puede valorar que dadas las características numéricas de las funciones de definición es siempre mucho más aconsejable dejar como parámetro libre a ajustar o a valorar predictivamente una tensión de trabajo en fluencia que la velocidad de deformación, o lo que es lo mismo, es mucho más precisa una predicción en tensión para el conformado que una predicción en velocidades de deformación.

Siendo el ajuste resultante obtenido con este método de alta significación, se constata que en un $78 \%$ de los valores usados para la predicción estos se encontraban dentro de las bandas de trabajo en las que se hallaban los valores experimentales de los puntos de la tabla I para el ajuste. En el caso de las representaciones normalizadas, necesarias para comparar con otras referencias bibliográficas, se constata que se puede garantizar con una fiabilidad del $95 \%$ una adecuada predicción en un orden de magnitud en velocidades de deformación con una imprecisión de un cuarto de orden. Estos valores son mucho mejores en cualquier caso en las predicciones para tensiones de trabajo.

\section{REFERENCIAS}

(1) Rieiro, I., Carsí, M. y Peñalba, F. Rev. Metal. Madrid, 32 (5), 1996: 285-356
(2) Rieiro, I., Carsí, M. y Peñalba, F. Comput. Methods and Testing for Engin. Integrity. Ed. T.V. Duggan. Southampton (R.U.), 1996: 301.

(3) RiEIRO, I. Tesis Doctoral. Madrid, 1997.

(4) Rieiro, I., Ruano, O.A., Eddahbi, M. y Carsí, M. J. Mater. Proc. Technol. 78, 1998: 177.

(5) Poirier, J.P. Creep of Crystals. Cambridge University Press. (R.U.), 1985.

(6) Poirier, J.J., Sotin, C. y Beauchose, S. Deformation process in minerals ceramics and rocks. Ed. Barber, D.J. and Meredith, P.G. Londres, 1990: 179-189.

(7) Sherby, O.D. y Burke, P. Mechanical behaviour of crystalline solids at elevated temperature. Prog. Mater. Sci. Vol. 3. Ed. Pergamon Press. Oxford (R.U.), 1968.

(8) Sellars, C.M. y Tegart, J.Mc G. Mem. Sci. Rev. Met., LXIII, (9), 1966: 731-746.

(9) LI, J.C.M. Acta Metall., 11, 1963: 1.269.

(10) Ruano, O.A. y Sherby, O.D. Rev. Phys. Appl., 23, 1988: 625-677.

(11) Luthy, H., Miller, A.K. y Sherby, O.D. Acta Metall., 28, 1979: 169-178.

(12) Garofalo, F. Joint. Int. Cont. on Creep. Londres, 1963: $1-31$.

(13) Canova, C.G., Shrivastava, S., Jonas, J.J. y Sellars, C.G. Formability of metallic materials-2000 A.D. ASTM. STP 753, 1982: 189.

(14) Barraclough, D.R., Whittaker, H.J., Nair, K.D. y SEllars, C.M. J. Test. Eval., 1 (3), 1973: 220-226.

(15) LaCh, E. y PöHLAndT, K. J. Mech. Working Technol., 9, 1984: 67-80.

(16) Fields, D.S. y Backoffen, W.A. Am. Soc. Test. Mater. Proc., 57, 1957: 1.259-1.272.

(17) Carsí, M., Peñalba, F., IbáÑez, J. y González-Doncel, G. Mater. Lett., 20, 1994: 119-123.

(18) Sheppard, T. y Norley, J. Mat. Sci. Technol., 4, 1988: 903.

(19) Sastry, S.M.L. et al. Proc. 4th Int. Conf. on Titanium. Jap. Inst. of Met. Kyoto (Japón), 1, 1980: 873. 